\title{
Bases de datos y revistas académicas en la pandemia de COVID-19: Imaginar futuros posibles para la comunicación científica después de la crisis
}

\author{
Databases and Academic Journals in the COVID-19 Pandemic: Imagining Possible Futures \\ for Scholarly Communication After the Crisis
}

\section{Bancos de dados e periódicos acadêmicos na pandemia COVID-19: Imaginando futuros possíveis para a comunicação científica após a crise}

Ivonne Lujano-Vilchis

Directory of Open Access Journals

Arizona State University

Arizona, Estados Unidos

ilujanov@asu.edu

(D) https://orcid.org/0000-0003-4245-8872

\begin{abstract}
Resumen: Se presentan algunas reflexiones sobre el trabajo del Directorio de Revistas de Acceso Abierto (DOAJ) durante el contexto de la pandemia de COVID-19, así como los desafíos para el futuro de las revistas de educación.
\end{abstract}

Palabras claves: Bases de datos; DOAJ; revistas académicas; COVID-19.

Abstract: Some reflections on the work of the Directory of Open Access Journals (DOAJ) during the context of the COVID-19 pandemic are presented, as well as the challenges ahead for education journals.

Keywords: Databases; DOAJ; scholarly journals; COVID-19.

Resumo: São apresentadas algumas reflexões sobre o trabalho do Directory of Open Access Journals (DOAJ) durante o contexto da pandemia COVID-19, bem como os desafios futuros para as revistas de educação.

Palavras-chave: Bancos de dados; DOAJ; Revistas acadêmicas; COVID-19.

Recibido: 28/09/2020 Aceptado: 09/10/2020 
https://doi.org/10.15359/ree.24-S.5

https://www.revistas.una.ac.cr/index.php/educare

educare@una.ac.cr

\section{Introducción}

Vivimos un momento de importantes retos para la comunicación científica y académica, especialmente por dos problemas que la pandemia por COVID-19 ha hecho evidentes: la falta de acceso a la información científica y, a su vez, el exceso de información poco relevante que circula en internet. Por un lado, a pesar de los avances en el acceso abierto, seguimos viendo que prevalece "la ciencia cerrada y el exceso de énfasis en las publicaciones de élite, sólo en inglés, independientemente del contexto y las consecuencias de la investigación" (Larivière et al., 2020). Por otro lado, esta es una de las "mayores explosiones de la literatura científica de la historia" (Brainard, 2020), pero muchas publicaciones sobre COVID-19 se tratan de especulaciones con fundamentos débiles e incluso propaganda (Kelly, 2020).

Por lo anterior, es muy pertinente el rol que ocupan las bases de datos académicas. Estas son útiles para "resolver la tensión entre el exceso de información y la escasez de capacidad de darle sentido" (Tenti Fanfani, 2020, p. 80), pues fungen como guía en la búsqueda de recursos avalados por las comunidades científicas. Me parece importante que la comunidad conozca el trabajo que realizan las bases de datos, por ello, en la primera parte de esta editorial comparto algunas experiencias del Directorio de Revistas de Acceso Abierto (Directory of Open Access Journals, DOAJ) durante la pandemia. Mi propósito es mostrar que una base de datos sostenida por la comunidad académica global es posible, incluso en tiempos convulsos. En la segunda parte, me sumo a las reflexiones de este suplemento especial sobre COVID-19 que publica la Revista Electrónica Educare. Para ello propongo pensar algunas preguntas sobre los retos que tienen las revistas en este contexto de crisis e incertidumbre para la educación.

\section{EI DOAJ durante la pandemia: la relevancia del apoyo de la comunidad global}

El Directorio de Revistas de Acceso Abierto es una de las bases de datos más utilizada en todo el mundo ya que contiene más de 15,000 títulos de revistas académicas. Todas las revistas listadas realizan revisión por pares y su contenido está disponible a través de sus páginas electrónicas, sin ningún costo por la consulta o descarga.

En los meses que han transcurrido desde el inicio de la pandemia, el trabajo dentro del DOAJ ha sido desafiante, en primer lugar, porque las solicitudes de indexación aumentaron con respecto a años anteriores. Durante el 2020 se han recibido en promedio 700 solicitudes cada mes, con el número más alto en el mes de junio: 940 solicitudes. Ello representa un reto en el proceso de aceptación de revistas, especialmente para las personas que realizan trabajo voluntario de revisión, su invaluable apoyo ha permitido procesar muchas de las solicitudes en tres meses (DOAJ, 2020a). 
https://doi.org/10.15359/ree.24-S.5

Más solicitudes de indización requieren un trabajo minucioso en la selección de revistas, para mantener un listado que se apega a los Principios de Transparencia y Mejores Prácticas en Publicaciones Académicas (Committee on Publication Ethics et al., 2013). Para apoyar esta tarea, DOAJ creó un Subcomité Editorial que brinda asesoría al Directorio sobre el proceso editorial y la calidad de los contenidos de las revistas, especialmente en casos que se requiere de observaciones de especialistas por disciplina (DOAJ, 2020c). Por ejemplo, en las revistas de informes clínicos, DOAJ adopta algunos criterios adicionales a los requisitos de indexación. Para que los informes de casos clínicos sean considerados como contenidos de investigación, deberán incluir el análisis retrospectivo de más de tres casos y/o una revisión de la literatura (DOAJ, 2020d). Si durante la revisión de solicitudes de indexación surgen dudas sobre este y otros tipos de contenidos, el Subcomité Editorial -conformado por profesoras y profesores voluntarios de instituciones ubicadas en Canadá, Estados Unidos, Filipinas, Finlandia, México y Reino Unido- asesora al equipo del DOAJ para tomar la decisión sobre la aceptación o rechazo de la solicitud.

Por otro lado, la discusión sobre la ciencia abierta se vuelve especialmente pertinente en el contexto de la pandemia por COVID-19, ya que implica un cambio en las prácticas de producción científica para "hacer que la investigación sea más abierta a la participación, revisión, refutación, mejora y reutilización para que el mundo se beneficie" (Bezjak et al., 2018). En este sentido, la ciencia abierta implica un cambio cultural (Fundación Karisma et al., 2018), por lo que será difícil su materialización en el corto tiempo. Sin embargo, para avanzar en ello, el DOAJ promueve algunas prácticas concretas, como la adopción de políticas editoriales partidarias de la reutilización de los materiales publicados. Por ejemplo, DOAJ recomienda el uso de algunas licencias Creative Commons, las cuales aseguran tanto a los autores como a las revistas la atribución de la autoría de sus artículos, permitiendo su reutilización y circulación libre en internet. En cuanto a los datos abiertos de investigación, DOAJ recomienda la adopción de políticas para la disponibilidad de los datos vinculados a los artículos. Además, el Directorio promueve la publicación de artículos sobre datos o conjuntos de datos. En este sentido, DOAJ ya incluye revistas que publican exclusivamente este tipo de artículos. En el contexto de una pandemia, el acceso a los datos es especialmente relevante para la reproducibilidad de la investigación.

Durante los últimos meses DOAJ ha establecido alianzas con diversas organizaciones para atraer al Directorio más revistas en idiomas distintos del inglés "como una forma de enfatizar la importancia de la investigación en todos los idiomas" (DOAJ, 2020b) y así contribuir a una distribución del conocimiento más equitativa y democrática. De esta manera el DOAJ se suma al compromiso de la Iniciativa Helsinki por el multilingüismo en la comunicación científica (Federation of Finnish Learned Societies et al., 2019). Un par de ejemplos son los acuerdos de colaboración con el proyecto Érudit en Canadá, así como con FinELib, Finnish Consortium for Universities. Futuras colaboraciones en este sentido incluyen algunos acuerdos con organizaciones en Latinoamérica, que se irán concretando en los próximos meses. 
https://doi.org/10.15359/ree.24-S.5

https://www.revistas.una.ac.cr/index.php/educare

educare@una.ac.cr

Para realizar todas estas labores, ha sido fundamental contar con el apoyo financiero de múltiples organizaciones en todo el mundo. Recientemente DOAJ recibió nuevas donaciones por parte de bibliotecas e instituciones académicas de Estados Unidos a través del Colorado Alliance for Research Libraries, así como de Arizona State University, San Francisco State University y Harvard University, y otras instituciones. Al mantener costos modestos de membresía para la financiación del DOAJ (una institución paga aproximadamente \$700 USD al año) se busca ampliar, lo más posible, la participación de la comunidad académica global. En América Latina, dos universidades colombianas, la Universidad Distrital Francisco José de Caldas y la Universidad Santo Tomás, han realizado donaciones anuales. Además, el Tecnológico de Monterrey (México) y la Sociedad Científica del Paraguay también han donado siguiendo el modelo SCOSS (The Global Sustainability Coalition for Open Science Services) con el cual las organizaciones se comprometen a brindar una aportación por tres años. Esta forma de financiamiento amplía la capacidad del DOAJ para servir a la comunidad académica mundial, y a la sociedad en general que, en tiempos tan convulsos, requiere de servicios eficientes para acceder a la información científica.

\section{Las revistas de educación frente a la pandemia, ¿qué futuros podemos imaginar?}

La pandemia implica nuevos desafíos para la investigación educativa. Ante el cierre de las escuelas y la "'domiciliación' de lo escolar" (Dussel, 2020, p. 1), entre otros fenómenos educativos que están surgiendo en medio de la crisis, investigadores e investigadoras de la educación nos encontramos con la responsabilidad de "elaborar explicaciones más precisas y alentar reflexiones que amplíen nuestras posibilidades de acción"(Estelles y Fischman, 2020, p. 2). Para ello, propongo preguntarnos sobre los medios de difusión académica que utilizamos, concretamente las revistas de educación: ¿nos ayudan a documentar adecuadamente el presente?, ¿estamos utilizando las revistas para conectar ideas y pensar futuros posibles para la educación después de la crisis?

Muchas personas investigadoras de la educación en todo el mundo se preguntan si la escuela volverá a ser la misma después de la pandemia, o si esta coyuntura desatará cambios sustanciales en los sistemas escolares. Quizá este sea un buen momento para preguntarnos también si las revistas de educación deberían seguir siendo las mismas. Cabría indagar si los procesos editoriales que hemos implementado hasta ahora seguirán siendo pertinentes para publicar sobre políticas educativas, teorías pedagógicas, modos de enseñanza, en tiempos donde es preciso "imaginar otras normalidades, más justas, menos desiguales, más sustentables" (Estelles y Fischman, 2020, p. 9). ¿Será entonces que precisamos de medios de comunicación académica que, además de difundir nuestros manuscritos, nos estimulen a imaginar esas otras normalidades? 


\section{Conclusión}

Propongo que como investigadoras e investigadores de la educación nos involucremos más en la reflexión sobre las revistas que necesitamos para diseminar nuestro trabajo en los años que vienen. Es necesario fortalecer las prácticas que han funcionado hasta ahora, como la distribución digital de nuestros manuscritos, pero también es preciso proyectar otros escenarios donde las revistas académicas sean aún más útiles para navegar nuevas crisis. Al final de cuentas, las escuelas y las revistas no son del todo distintas: ambas son lugares de encuentro, generadoras de diálogos, constructoras y promotoras de saberes. Ambas deben responder a su tiempo, y especialmente en este tiempo requerimos fortalecerlas para conversar ahí sobre las transformaciones que necesitamos para evitar colapsos mayores, los cuales pueden ser incluso más graves que esta pandemia.

Nota general: La traducción y corrección de estilo han sido realizados por la autora.

\section{Referencias}

Bezjak, S., Clyburne-Sherin, A., Conzett, P.; Fernandes, P., Görögh, E., Helbig, K., Kramer, B., Labastida, I., Niemeyer, K., Psomopoulos, F., Ross-Hellauer, T., Schneider, R., Tennant, J., Verbakel, E., Brinken, H. y Heller, L. (2018). Open Science Training Handbook. Zenodo. https://doi.org/10.5281/zenodo.1212496

Brainard, J. (2020). Scientists are drowning in COVID-19 papers. Can new tools keep them afloat? Science |AAAS. https://doi.org/10.1126/science.abc7839

Committee on Publication Ethics, Directory of Open Access Journals, Open Access Scholarly Publishers Association y World Association of Medical Editors (2013). Principles of transparency and best practice in scholarly publishing. https://publicationethics.org/ $\underline{\text { node/39286 }}$

DOAJ. (2020a). COVID-19: Turnaround times may be affected. DOAJ News Service. https://blog. doaj.org/2020/04/01/COVID-19-turnaround-times-may-be-affected/

DOAJ. (26 de mayo, 2020b). A partnership between Érudit and DOAJ: Towards greater visibility and discoverability for open access French-language and bilingual scientific journals. DOAJ News Service. https://blog.doaj.org/2020/05/26/a-partnership-between-erudit-anddoaj-towards-greater-visibility-and-discoverability-for-open-access-french-languageand-bilingual-scientific-journals/

DOAJ. (10 de julio, 2020c). Introducing the Editorial Subcommittee. DOAJ News Service. https:// blog.doaj.org/2020/07/10/introducing-the-editorial-sub-committee/ 
https://doi.org/10.15359/ree.24-S.5

https://www.revistas.una.ac.cr/index.php/educare

educare@una.ac.cr

DOAJ. (26 de agosto, 2020d). Seal criteria and additional criteria for some journal types. DOAJ News Service. https://blog.doaj.org/2020/08/26/seal-criteria-and-additional-criteria-forsome-journal-types/

Dussel, I. (2020). La escuela en la pandemia. Reflexiones sobre lo escolar en tiempos dislocados. Práxis Educativa, 15, 1-16. https://doi.org/10.5212/PraxEduc.v.15.16482.090

Estelles, M.y Fischman, G. E. (2020). Imaginando una educación para la ciudadanía global después del COVID-19. Práxis Educativa, 15, 1-14. https://doi.org/10.5212/PraxEduc.v.15.15566.051

Federation of Finnish Learned Societies, Committee for Public Information, Finnish Association for Scholarly Publishing, Universities Norway y European Network for Research Evaluation in the Social Sciences and the Humanities. (2019). Iniciativa Helsinki sobre multilingüismo en la comunicación científica. helsinki-initiative.org. https://www.helsinki-initiative.org/es

Fundación Karisma, Centro de Internet y Sociedad de la Universidad del Rosario y Sistema de Información para la Biodiversidad de Colombia. (2018). Declaración de Panamá sobre Ciencia Abierta -Foro CILAC. http://forocilac.org/declaracion-de-panama-sobre-cienciaabierta/

Kelly, É. (25 de junio, 2020).COVID-19 pandemic leads to flood of'useless'science. Science|Business. https://sciencebusiness.net/COVID-19/news/COVID-19-pandemic-leads-flood-uselessscience

Larivière, V., Shu, F. y Sugimoto, C. R. (5 de marzo, 2020). The Coronavirus (COVID-19) outbreak highlights serious deficiencies in scholarly communication. LSE [Mensaje en un blog]. https://blogs.Ise.ac.uk/impactofsocialsciences/2020/03/05/the-coronavirus-COVID-19outbreak-highlights-serious-deficiencies-in-scholarly-communication/

Tenti Fanfani, E. (2020). Educación escolar post pandemia. Notas sociológicas. En I. dussel, P. Ferrante y D. Pulfer (comps.), Pensar la educación en tiempos de pandemia. Entre la emergencia, el compromiso y la espera (pp. 71-83). UNIPE Editorial Universitaria. https:// abacoenred.com/wp-content/uploads/2020/08/pensarlaeducacion.pdf 